\title{
Neuroimaging: Overview of Methods and Applications
}

\author{
Lee Ryan, Ph.D. ${ }^{1,3}$ and Gene E. Alexander, Ph.D. ${ }^{2,3}$
}

${ }^{1}$ Cognition and Neuroimaging Laboratories, Departments of Psychology and Neurology, University of Arizona, Tucson

${ }^{2}$ Neuroimage Analysis Laboratory, Department of Psychology, Arizona State University, Tempe and ${ }^{3}$ the Arizona Alzheimer's Disease Consortium, AZ

2007

In L. Luecken and L. Gallo, Eds., Handbook of Physiological Research Methods in Health Psychology. Elsevier.

Corresponding author: Gene E. Alexander, Ph.D., Department of Psychology, Arizona State University, Tempe, AZ 85287; phone: 480-727-7790; fax: 480-965-8544; email: gene.alexander@asu.edu 


\section{Introduction}

The past few decades have seen enormous advances in the ability to visualize and measure aspects of brain anatomy and physiology in the living human being. The recent developments in neuroimaging techniques that provide measures of brain structure and function, as well as cerebral activity during cognitive processes such as perception, attention, and memory have changed the face of cognitive neuroscience, and have allowed us to ask questions about the relation between the brain and behavior that were simply not possible only a decade or two ago. As these techniques have become more accessible and affordable for research use, they have been increasingly applied to a variety of research questions, having the potential to greatly enhance our understanding of the normal brain and its development throughout the lifespan. Even areas of research that have traditionally not been linked to neuroscience, such as social psychology, economics, and education are increasingly making use of neuroimaging methods.

From the beginning, evaluating the effects of health-related factors, such as aging, neurological and psychiatric illness, and brain injury have been an important and rapidly growing part of the neuroimaging field. Health psychology provides a potentially unique perspective in the application of neuroimaging methods studying how brainbehavior relationships are altered by disease states and other health-related factors. Neuroimaging provides a set of tools that can address a wide variety of questions. For example, researchers might address how neural systems important for specific cognitive processes are altered by the presence, progression, and treatment of a disease; test the effect of health factors such as genetic risk on regional patterns of 
brain structure and function; identify subgroups for which treatment or prevention therapies are most effective; or determine the effect of individual differences on the expression, severity, and progression of brain diseases. To utilize these techniques effectively, however, those new to the neuroimaging field and unfamiliar with its rapidly evolving technology are often faced with the daunting task of figuring out where and how to begin.

The purpose of this chapter is to provide an overview of the major neuroimaging methods and their applications to health psychology research. Since a detailed and complete description of the methods and general applications for neuroimaging in health-related research is well beyond the scope of this chapter, we have focused on the description of methods and applications that we believe will provide a useful starting point for researchers who are thinking of applying such neuroimaging techiques to their own areas of health-related psychology.

We begin with a brief review of the major structural and functional neuroimaging research methods that are commonly in use, including computed tomography (CT), magnetic resonance imaging (MRI), positron emission tomography (PET), functional magnetic resonance imaging (fMRI), and diffusion-weighted magnetic resonance imaging (DWMRI). This section will include a brief history of neuroimaging, followed by a description of the origins of the signal that is measured by each method and a discussion of the major benefits and limitations associated with each approach. We will also address general issues and approaches for analysis of neuroimaging data, including a discussion of the use of univariate and novel multivariate methods. 
Following our review of the major neuroimaging methods, we will illustrate how these neuroimaging techniques can be applied to health-related issues, focusing our discussion on applications to the study of aging and the most common form of agerelated dementia, Alzheimer's disease. Finally, we will present a list of suggested readings and websites that provide additional resources and information for those who would like to begin to use these methods.

\section{History of brain imaging}

Imaging the brain's structure

The English scientist Godfrey Hounsfield used a computer to successfully combine a series of conventional X-ray images taken at multiple angles around the head, producing cross-sectional brain images of unprecedented clarity. The first clinical results using X-ray computed tomography (CT) were reported in 1972 (Ambrose \& Hounsfield, 1972a, b), changing overnight the way in which we look at the human brain. Until then, the common method available for brain imaging was also based on X-ray imaging. This method, referred to as pneumoencephalography, was performed by draining cerebrospinal fluid from spaces in and around the brain and replacing it with air, oxygen, or helium to allow the structure of the brain to show up more clearly on conventional X-ray. Although this procedure continued to be performed until the late 1980 's, it was painful and posed significant risks for patients. This procedure was supplanted by the CT scan, which could produce higher quality brain images safely and non-invasively. Hounsfield's work on CT not only provided a superior brain imaging method, but also a mathematical technique (tomography or back-projection) where 
digital geometric processing is used to generate a three-dimensional image of the internal structure of the brain from a large series of two-dimensional images, and would provide a foundation for the three dimensional reconstruction method used in many imaging methods that followed.

Magnetic resonance imaging (MRI) emerged contemporaneously with $\mathrm{CT}$, but had its roots in the field of nuclear magnetic resonance (NMR). The MRI signal is based on principles of the movement of hydrogen atoms or protons in a magnetic field which were described independently by physicists Felix Bloch (1946) and Edward Purcells (1946) in the 1940's. Their experiments, with a sample of a chemical compound placed in a strong magnetic field, a transmitter coil that sent electromagnetic energy to the sample, and a detector coil that measured energy emitted back from the sample, forms the basis of all modern magnetic resonance imaging. For their work, Bloch and Purcells were jointly awarded the Nobel Prize in Physics in 1952. For many years, NMR's primary application was spectroscopy, or the chemical analysis of samples.

In the early 1970's, the American chemist Paul Lauterbur (1973) applied NMR principles to create a two-dimensional image of an object. He determined that if the strength of the magnetic field was varied over the space occupied by an object, the resonant frequency of protons at different spatial locations would also vary. By measuring how much energy was emitted at different frequencies, one could identify how much of that object was present at each spatial location. Lauterbur's imaging method was soon made more efficient by using echo-planar imaging, proposed by Peter Mansfield in 1976, in which a complex signal is acquired that derives from all aspects of 
the object simultaneously. The resulting complex signal could be reconstructed into separate spatial locations using Fourier analysis techniques. Taken together, these techniques provide the basis for modern anatomical and functional MRI. Lauterbur and Mansfield were both awarded the 2003 Nobel Prize in Physiology or Medicine for their work.

In the course of developing human imaging, the term NMR was abandoned in favor of MRI, in large part because of the negative health connotations of the word nuclear, which was especially justified because MRI does not employ ionizing radiation. General Electric produced the first commercially-available human-body 1.5T scanner in 1982. Other manufacturers followed and the 1.5T scanner has since been the standard for clinical use. The FDA approved MRI scanners for clinical use in 1985, opening the door for the use of MRI as a diagnostic tool that could be prescribed by physicians for clinical use and billed to insurance companies for reimbursement.

Although CT was already being used quite widely in clinical settings, the diagnostic value of MRI quickly became apparent because of its superior ability to differentiate tissue types, rather than just gross anatomy. The earliest major impact of MRI was in the detection of demyelinating lesions in patients with multiple sclerosis. The first MRI clinical case series in ten patients with multiple sclerosis came from Hammersmith Hospital, London (Young et al., 1981). CT scans were performed with and without contrast agents, identifying a total of 19 white matter lesions. MRI scans on the same patients revealed not only these 19 lesions, but an additional 112 lesions. The implications for MRI as a sensitive marker in the early detection of multiple sclerosis quickly emerged. Whereas CT continues to play a very important role in 
neuroimaging, MRI methods have further evolved to provide exquisite contrast sensitivity for many types of pathological brain tissue, including tumors, stroke, demyelination, and infection.

Physiological and functional imaging of the brain

Interest in measuring biochemical and physiological processes within the brain goes back at least to the late 1800's. Paul Broca, the French neurologist best known for his observations on the effect of left frontal lesions on language function, was also interested in the notion that blood flow changes were associated with mental activities (Broca, 1879). Although appropriate methods to assess local brain activity were not yet available, the general notion of the connection between circulation and brain function, and the interest in identifying an unbiased method of measuring changes in brain function in response to cognitive activity was an important early contribution.

Electrical signaling by neurons in the brain is a metabolically active process that increases the demand for oxygen and for glucose, the major source of energy for neurons. It followed, then, that cerebral blood flow (rCBF), which transports oxygen and essential nutrients including glucose to the brain, might provide an index of functional brain activity (Sokoloff et al., 1982; Baron et al, 1984).

Kety and Schmidt (1945) were the first to implement a measure of brain blood flow. Although the Kety-Schmidt technique was the first truly quantitative method for measuring whole brain blood flow in humans, it did not provide regional brain measurements and it was highly invasive, requiring internal jugular vein and arterial blood sampling. Soon afterwards, Lassen and colleagues (1963) introduced a less 
invasive method that measured regional blood flow using external scintillation crystal detectors arrayed around the surface of the head. A freely diffusible radioactive gas, such as xenon-133, was injected into the patient (or, in later developments, inhaled), and the detector array measured the distribution of the gas as it perfused the brain, creating a two dimensional image of blood flow. These methods provided the first clear evidence in humans that regional blood flow changes correlated with changes in brain activity (Obrist et al., 1967; Risberg, 1980; Prohovnik, 1988), and have been used subsequently to show regional brain reductions in patient groups (Prohovnik et al., 1989; Alexander et al., 1995) and regional differences in blood flow associated with cognitive performance (Alexander et al., 1994). The limited spatial resolution of these two-dimensional rCBF images, as well as the inability to directly assess activity from deeper subcortical brain structures, led to further advances, such as the application of tomographic reconstruction to create 3-D images and the invention of other radioactive tracers that more directly assess metabolism in the brain. These methods provide the basis for positron emission tomography (PET).

PET creates images of brain metabolic function based on autoradiographic techniques for measuring glucose metabolism in laboratory animals (Sokoloff et al., 1982). Since the energy needed for the electrical activity of neurons in the brain is provided by glucose, measuring the utilization of glucose in the brain provides a direct index of neural activity. In PET, a radioactively labeled form of glucose is injected into the patient, and the uptake of the labeled glucose into various brain regions is measured (Sokoloff et al., 1982). Like the rCBF images described above, PET images are acquired using circular arrays of detectors that accumulate images at various angles 
around the head, and are reconstructed using computer tomography techniques to produce 3D images similar to those developed for CT. With advances in scanner resolution (Jagust et al., 1993), methods for quantification (Frackowiak et al., 1980), and the development of radioactive tracers to measure rCBF, regional cerebral metabolic rates for glucose $(\mathrm{rCMRglc})$ and oxygen $\left(\mathrm{rCMRO}_{2}\right)$, it became possible to assess patterns of regional brain activity that are present in mental resting state or while an individual is performing various cognitive tasks (Posner \& Raichle, 1994). In addition to measuring functional brain changes during mental activity, PET can also be used to measure a variety of normal and pathological brain processes. The development of other physiologically relevant radiolabled compounds provides the ability to measure, for example, the uptake of specific neurotransmitter precursors, neuroreceptor density and binding, aspects of cellular processes such as signal transduction, and the distribution of various types of neuropathology (Busatto and Pilowsky, 1995; Rapoport, 2005; Cagnin, et al., 2001; Klunk, et al., 2003).

Another method of imaging brain activity, functional magnetic resonance imaging (fMRI), emerged in the late 1980's, when it was observed that changes in neuronal activity resulted in local changes in the amount of oxygen in tissue (Fox \& Raichle, 1986). Ogawa and Lee (1990) determined that changing the amount of oxygen carried by hemoglobin in the blood changes the degree to which hemoglobin disturbs a magnetic field. Further, they demonstrated that these in vivo changes in blood oxygenation in response to neuronal activity could be detected with MRI as a subtle increase in signal strength, and that this signal change was regionally localized in brain. The technique became known as the Blood Oxygen Level Dependent (BOLD) method, 
and researchers soon demonstrated the utility of fMRI for detecting and localizing functional brain activity in humans during the performance of cognitive tasks (Bandettini et al., 1992). The method has the distinct advantage that it is non-invasive, since it does not require the use of radioactive tracers or other contrast compounds. In the few years since its inception, fMRI has proven to be an exceptionally useful tool for assessing brain function, and has already made an enormous impact on the field of cognitive neuroscience.

\section{Methods}

Imaging brain anatomy

\section{Computerized tomography (CT)}

CT can be a very useful tool in research, providing a method for the visualization of anatomical structures and abnormalities with high resolution. CT images use X-ray refraction to highlight the contrast between brain tissues of differing densities. CT scanners transmit a finely collimated beam of X-rays that moves around the patient's head within a metallic shell called a gantry. The degree to which X-rays are absorbed by the head is measured with an array of scintillation detectors that determine the attenuation of the X-rays as they pass through tissues of different densities. Using tomographic and back-projection computer algorithms, cross-sectional images of the brain are reconstructed in which each element of the image, or pixel, is allocated a level of brightness somewhere between black and white, usually with a scale of 256 shades of gray. The image thus reflects the density of tissue at a given location. Generally, tissues with high radiation attenuation, such as bone, appear white, whereas 
cerbrospinal fluid, which has low attenuation, appears black. The brain has intermediate levels of attenuation and appears gray.

CT thus provides good visualization of the boundaries between cerebral tissue and the ventricles. It permits identification of a wide range of cerebral abnormalities, including the presence of space-occupying lesions and shifts in midline brain structures, cerebral infarction and hemmorhage, and demyelination. It is useful in tracking atrophy and for identifying certain specific types of tumors. Iodinated contrast agents can also be administered intravenously with CT to enhance differences in tissue density, which can help identify vascular abnormalities and the breakdown of the blood brain barrier, which may occur in certain types of tumors, infections, and other inflammatory processes.

CT has several advantages for research. First, it is widely available and relatively inexpensive compared to MRI. It can also produce images with relatively high spatial resolution quickly, so that patients who cannot tolerate long procedures can usually be scanned with CT. Another important advantage of CT over MRI is that it doesn't require people to be in an enclosed space, so that the approximately $10 \%$ of people who find themselves having claustrophobic reactions to MRI can tolerate CT quite well. It is also a good choice for patients in which MRI is contraindicated (e.g., with cardiac pacemakers and metallic implants). The main drawback of CT is the presence of beam-hardening artifacts that make it difficult to visualize brain tissue in proximity to bone, such as brain regions near the base of the skull (e.g., orbitofrontal cortex). It also has relatively poor contrast between gray and white matter in the brain, making it less useful for quantifying volumes of gray and white tissue compartments 
separately, and it is relatively insensitive to white matter hyperintensities which can reflect microvascular abnormalities related to cardiovascular risk factors and other health conditions. This sensitivity increases considerably with the use of iodinated contrast, but the potential for severe allergic reactions to iodine for some individuals is a significant concern. Nevertheless, for clinical evaluations to provide screening for research subjects and for global measures of brain volume or abnormality, CT can be a very useful and economical research tool.

\section{Magnetic resonance imaging (MRI)}

MRI has become an essential tool in evaluating neurological disease, and more recently, in providing various measurements of brain physiology and function. Human brain tissue contains significant amounts of water, proteins, lipids, and other macromolecules, which contain abundant hydrogen atoms. MRI relies on the fact that the nuclei of hydrogen atoms contain positively charged particles or protons which spin around their own internal axis. A fundamental feature of electromagnetism is that a moving charge creates it's own small magnetic field, so the spinning protons are essentially small dipoles. When placed in a strong external magnetic field, the protons inside the body align along the direction of the strong magnetic field. When a radiofrequency pulse is applied that matches the spin frequency of hydrogen (the Lamor frequency), some of the protons aligned with the magnetic field will absorb this energy and flip over, reversing their direction. Once the radiofrequency pulse is turned off again, the protons "relax" back to their original orientation, emitting the absorbed energy once again, which can be measured with a simple radiofrequency receiver. The 
strength of the signal that is emitted is directly proportional to the number of protons in the magnetic field.

The components of an MRI scanner include a magnet to produce a strong, uniform field, a radiofrequency system to transmit and receive signals, a gradient magnet system for spatial encoding, and a computer system to control the process and to reconstruct the images. The typical clinical MRI magnet has a field strength of 1.5T (magnetic field strength is measured in units of Tesla, or T), which is approximately 30,000 times greater than the magnetic field of the earth. Currently the FDA approves scanners up to 3T for human clinical purposes, although higher field strengths are used in some sites for human research. The main magnetic field is created by a superconducting magnet, essentially a large coil of wire with electrical current running through it, cooled to a temperature of $4 \mathrm{~K}$ (-269 degr C) using liquid helium. At these temperatures, the wires are in a superconducting state, having no electrical resistance. A set of shim or gradient magnets are placed within the main coil, which are used to produce the linear field gradients that allow spatial localization of the signal. Fourier transformation is used to analyze the complex mixture of signals, determining the intensity of the signal at each $x-y-z$ coordinate, thus creating a set of two-dimensional images of the head. One gradient coil is used to select only a particular slice or brain section (in the $z$ axis) so that only the protons within that slice absorb the energy in the radiofrequency pulse. Two other gradient coils are used to determine the $x$ and $y$ coordinates of a point within the slice from which the signal originates. The amplified signal obtained from the radiofrequency receivers is then transferred to a computer for image reconstruction. 
The rate at which protons relax to their original orientation is determined by two parameters - T1 (the spin-lattice or longitudinal relaxation) and T2 (the spin-spin or transverse relaxation). Importantly, T1 and T2 relaxation rates differ for different types of brain tissue, and these properties can be used to produce images that highlight the contrast between particular tissue types. The visual contrast or intensity differences between tissue types of a particular MRI scan are therefore under the direct control of the individual operating the scanner console and depend on the timing parameters of the imaging, including the time between successive radiowave pulses (TR), the time between excitation and signal sampling (TE), and the strength and number of radiofrequency pulses applied. This makes MRI an exceptionally versatile imaging method for diagnostic and research purposes.

Every image in MRI is weighted with both $\mathrm{T} 1$ and $\mathrm{T} 2$, as well as by protondensity weighting, or the sheer number of hydrogen protons at a given spatial location. For example, tumors and other tissues with a large amount of freely moving water usually appear dark in T1 weighted images, but bright in proton-density or T2 weighted images. In general, T1 weighted images give a sharper definition of gray and white matter boundaries which can be important for quantifying gray or white matter atrophy and rates of volume change in specific brain structures, while T2 weighted sequences are more sensitive to the certain types of pathologies, such as identifying the presence and size of tumors or cerebrovascular abnormalities. Most clinical sequences use all three types of images, i.e., T1 weighted, T2 weighted, and proton density weighted. Sometimes, a contrast agent is used that increases contrast sensitivity for specific pathologies. Gadolinium, a rare earth element, is a paramagnetic substance that 
readily binds to vascular compartments, and can highlight locations where the blood brain barrier has deteriorated, as is commonly seen in pathological states such as tumors, metastases, and multiple sclerosis. For diagnosis, MRI is generally considered clinically superior to CT in detecting edema, cysts, some types of tumors, the vascular system, hemorrhage, and damage to white matter. It is important to note that clinical patients and research subjects must be carefully screened for possible contraindications for MRI before being placed in the scanners high magnetic field, including the presence of metal implants (such as joint replacements, aneurysm clips, cochlear implants), cardiac pacemakers, and shrapnel. Figure 1 shows an example of the same section of a brain MRI acquired with proton density, $\mathrm{T} 1$, and $\mathrm{T} 2$ weighting.

Figure 1: Example of MRI scans using proton density, T1, and T2 weighted sequences. The same axial slice is shown for the three image sequences obtained from a single healthy, young adult. The cerebrospinal fluid in the ventricles are indicated by the white areas in the center of the T2 weighted image and by the corresponding black areas in the T1 weighted scan.

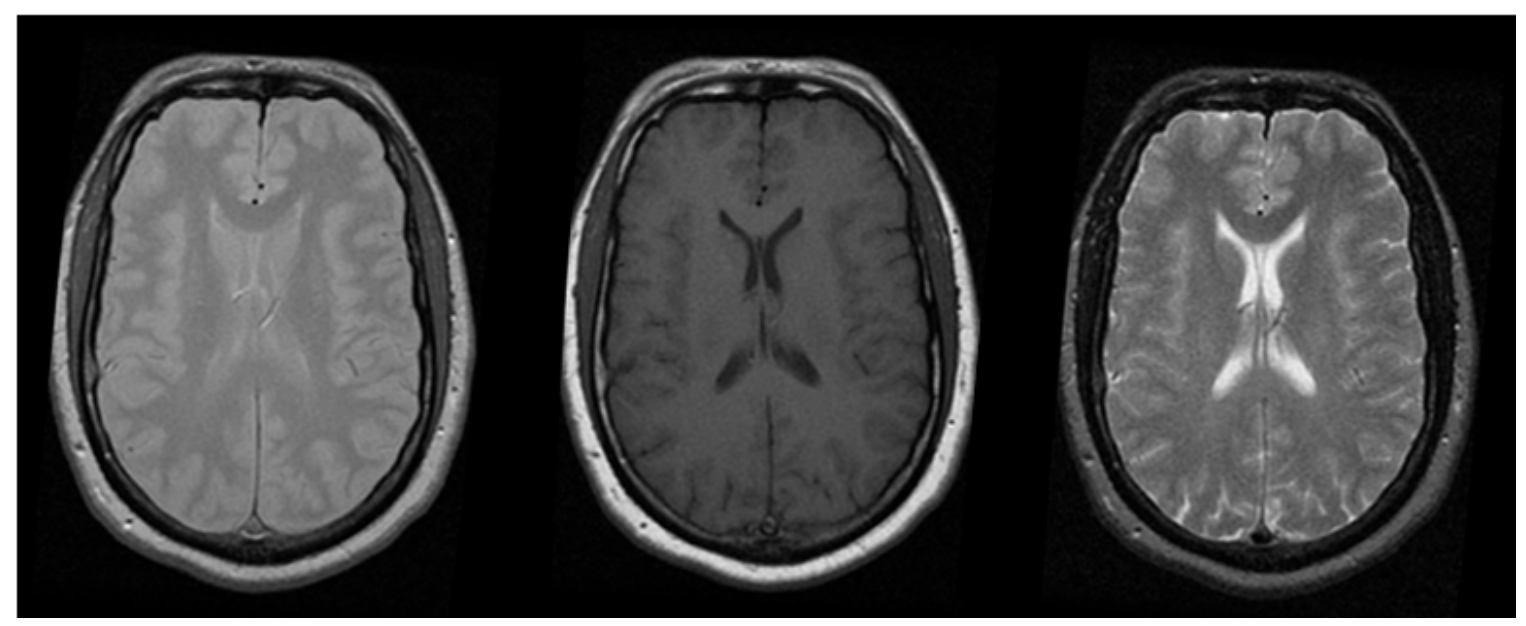

Proton-density

T1-weighted

T2-weighted 
Imaging the function of the brain

Positron emission tomography (PET)

In PET, positron-emitting radiotracers are used to construct functional images of the brain's physiological and metabolic response to neural activity. PET radiotracers are compounds that bind to a particular molecule of interest (for example, oxygen, glucose, or others) which is utilized by the brain. These radiotracers contain excess protons in the nucleus and thus are unstable. A short time after the bound molecule is taken up by brain tissue, the tracer will break down, emitting positrons (electrons with a positive charge) that can travel a few millimeters within tissue. When it is nearly at rest, the positron then collides with an unbound electron causing an annihilation event (i.e., where the positively and negatively charged electrons meet). This results in two highenergy gamma rays that travel away from the annihiliation event at exactly 180 degrees apart. These "events" can be detected by a gamma ray camera that is outfitted with sodium iodide crystal detectors arrayed in a ring 360 degrees around the head. Using pairs of detectors situated 180 degrees apart, the twin gamma rays are selected only if they strike the oppositely positioned detectors at almost the same instant, and thus a cross-sectional image is formed from the detection of the annihilation events. With the use of tomographic reconstruction algorithms, a 3-D image of the distribution of radioactivity can be created, which in turn indicates where metabolic activity has occurred in the brain.

Positron-emitting radiotracers (also referred to as radionucleides) used in PET imaging are available that are bound to a number of important biological elements, 
including carbon, oxygen, nitrogen, iron, and fluorine. The most widely used tracer for studies of functional brain activity with PET is F18-labeled deoxyglucose or the FDG method. The FDG tracer is most often used to assess cerebral glucose metabolism in a resting state (e.g., with eyes closed and ears covered) to evaluate regional patterns of reduced brain function. FDG has been used to aid diagnosis and to track changes in regional brain activity over time or in response to treatment (e.g., Silverman et al., 2001; Alexander et al., 2002). Figure 2 shows the regions of reduced glucose metabolism typically observed in FDG PET group studies of AD patients compared to healthy controls. After intravenous injection, FDG enters the brain where it allows for regional glucose utilization to be measured. Oxygen-15 labeled water $\left(\mathrm{H}_{2}{ }^{15} \mathrm{O}\right)$ is also widely used to determine cerebral blood flow and volume. Since $\mathrm{H}_{2}{ }^{15} \mathrm{O}$ decays rapidly with a half-life of about 2 minutes, this tracer has been used extensively for assessing neural activity during cognitive activation studies where repeated measurements under different experimental conditions are often needed in the same session. With the development and availability of fMRI, the use of PET for normal cognitive activation studies has greatly diminished. 
Figure 2: Group comparison of patients with Alzheimer's disease (AD) and healthy controls on a voxel basis using 18-FDG PET acquired in a resting state with eyes and ears covered. Regional differences are mapped on lateral and medial projections for the right and left hemispheres. Lower glucose utilization $(p<.001)$ in the AD patients than controls is indicated in parietal, temporal, occipital, and frontal brain regions by the gray scale with the light regions showing the most significant group differences. (Adapted from Alexander et al., American Journal of Psychiatry, 2002)

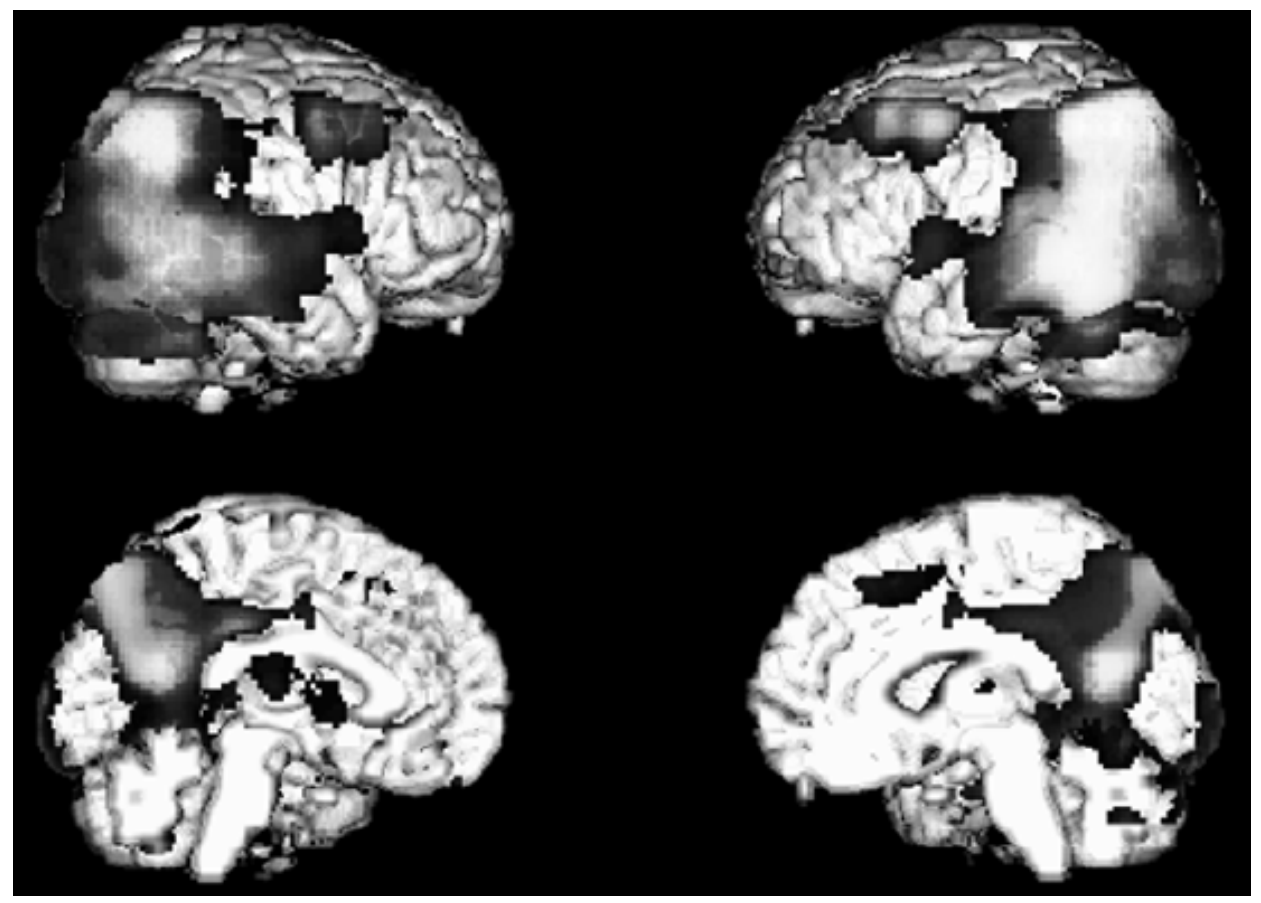

In contrast, PET currently offers more flexibility than fMRI for monitoring the activity of many different molecules in the brain. For example, PET has been used to measure the regional distributions of dopamine receptors and dopamine uptake, opiate receptor binding of various pharmacological agents, and more recently cellular processes such as signal transduction and specific pathological targets (Busatto and Pilowsky, 1995; Rapoport, 2005; Cagnin, et al., 2001; Klunk, et al., 2003).

PET facilities require a cyclotron to produce the radiotracers, and because most of these tracers decay quickly, the cyclotron typically must be located onsite with the PET camera system. In addition, a group of radiochemists must be available to provide the variety of radiotracers within pharmaceutical quality standards and in suitable 
amounts for safe and effective human use. Thus, while the availability of PET imaging for clinical and research use has grown considerably in recent years, the space requirements and high cost of operation for a fully equipped PET facility has been a limitation in its widespread use for research outside of major medical centers.

\section{Functional magnetic resonance imaging (fMRI)}

In fMRI, regional neural activity is assessed by measuring changes in oxygen content of the blood (Ogawa and Lee, 1990). This indirect measure of brain function is referred to as the "hemodynamic response" and provides the basis for the imaging signal that is used to determine regional brain activity with fMRI.

The technique has been used extensively to provide information on regional brain changes that occur in response to manipulations of cognitive or behavioral task conditions and has more recently been used to assess regional cerebral blood flow and blood volume during baseline resting states. In fMRI, specialized MRI hardware is used that allows ultrafast imaging. fMRI relies on an "endogenous" contrast agent, where a naturally occurring paramagnetic substance, deoxygenated hemoglobin, alters the homogeneity of the local magnetic field, resulting in a localized loss of signal. In response to neural activity, local metabolic need of the brain tissue increases, resulting in increased regional blood flow that delivers oxygen-rich hemoglobin. Because the oxygen delivered actually exceeds the demands of the tissue in the region, there is a short-lived net increase in oxygenated hemoglobin and a decrease in deoxygenated hemoglobin. The change in the ratio of paramagnetic deoxygenated hemoglobin to 
oxygenated hemoglobin results in a small but measurable increase in MR signal that lasts for several seconds.

The resulting net decrease in deoxyhemoglobin concentration in the blood and a net increase in signal is commonly referred to as the Blood Oxygenation Level Dependent (BOLD) effect. Importantly, these changes in oxygenation in response to increased cognitive demands are highly localized, so that the change in signal can be used to infer the specific region of brain that is involved in a particular cognitive process, within the limits of the spatial resolution of the scanner.

The first fMRI paper demonstrating its use in measuring activity during a cognitive task was published in the early 1990's (Bandettini et al., 1992). Despite being the relative newcomer in the neuroimaging field, fMRI has seen an explosion of interest and applications in research that has enhanced our understanding of the neural systems underlying normal cognitive function and has been increasingly applied to studies of the effects of pathological conditions such as Alzheimer's disease, stroke, schizophrenia, and depression. An example of an fMRI brain map and voxel timeseries obtained during a visual task is shown in figure 3 . 
Figure 3: Percent fMRI signal change over a $12 \mathrm{sec}$ period after a simple button-press response to a visual stimulus (aligned at 0 secs). Time-series data are presented for a single voxel (right panel) identified by the crosshair on the brain map (left panel), averaged across $200+$ trials. Regions of significant activation are depicted on the brain image in gray $(p<.001)$ and white $(p<.0001)$.
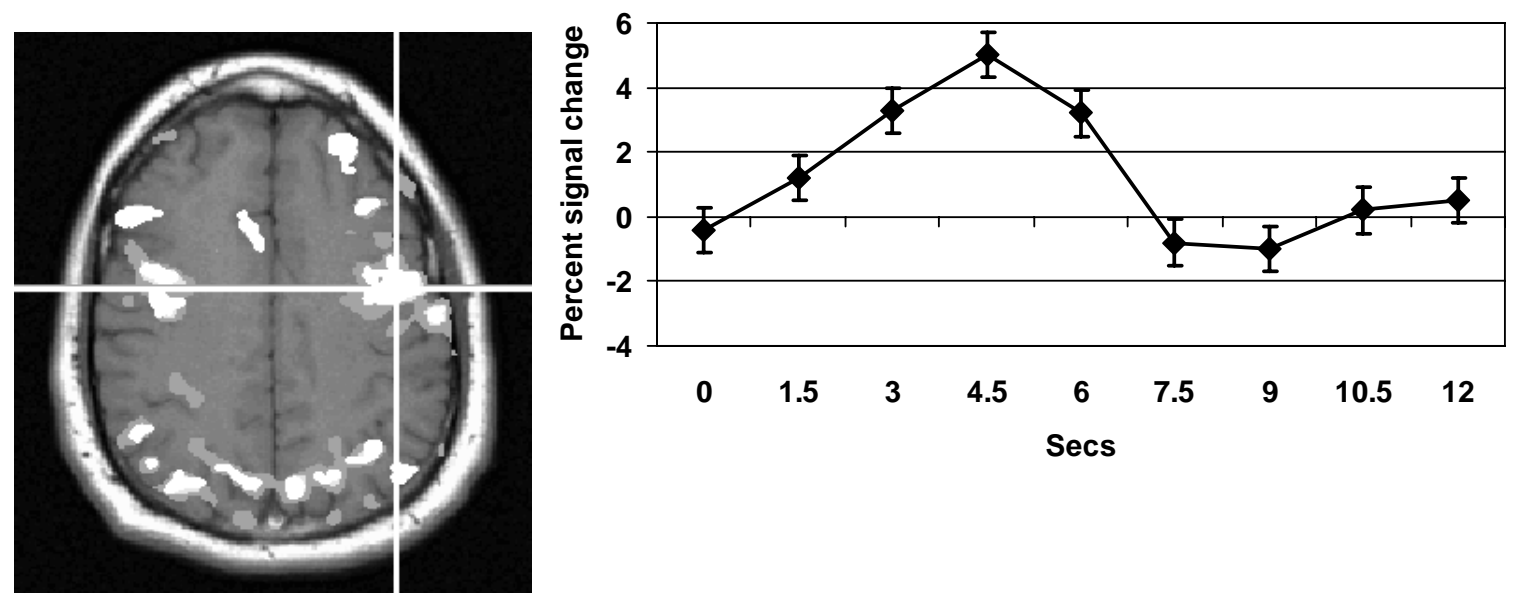

Advantages of $\mathrm{fMRI}$ include its high spatial and temporal resolution relative to PET, and the ability to acquire images quickly. The non-invasive nature of fMRI, in particular the absence of ionizing radiation that is present in PET, allows for frequent repeated measurements, making within-subject multi-session studies practical. fMRI can be conducted on any clinically-available scanner with the appropriate hardware, and most manufacturers now provide fMRI pulse sequences and basic analysis software on their scanners as standard equipment, greatly enhancing accessibility for researchers and clinicians alike.

There are, however, a number of limitations in the use of fMRI that need to be considered when designing research studies. Head movement during the scanning session is an important source of artifact and a potential problem for research studies, particularly when working with populations such as young children or patients who may find it difficult to remain motionless during the extent of the imaging session. Approximately 10 percent of individuals have claustrophobic or anxiety reactions due to 
the confined space in the bore of the magnet. All participants must be carefully screened for contraindications to MRI, which would include the presence of metal implants (e.g., joint replacements, aneurysm clips, cochlear implants), cardiac pacemakers, and shrapnel, which collectively can remove a significant number of potential participants from the experimental subject pool. Because images are typically collected very quickly, approximately every $30 \mathrm{~ms}$, fMRI images have problematic spatial distortion and signal loss artifacts in areas of the brain that are adjacent to air, such as the anterior temporal lobes adjacent to the sinuses, the lateral temporal lobe near the ear canals, and the orbital frontal cortex. In addition, there are many practical limitations to working with computers and other equipment for cognitive task presentation in the high field strength magnetic environment. Specialized equipment designed specifically to work in such magnetic field environments must be used in order to present experimental stimuli to participants in the scanner and also to gather behavioral responses.

Despite the limitations, with proper training and knowing the risks and pitfalls, fMRI methods have proven to be a very safe and effective research tool that has greatly enhanced our understanding of the neural systems involved in cognition and behavior.

\section{Diffusion-weighted MRI}

A recently developed MRI-based method is proving particularly useful in identifying inflammation and the integrity of white matter structures within the brain. The method, known as diffusion-weighted magnetic resonance imaging (DWMRI), measures the Brownian motion of water molecules as they diffuse normally within and across 
compartments. Several measurements can be obtained, including quantitative measures of the total diffusivity of water in a voxel, the degree to which that movement is directional (referred to as anisotropy), and the actual direction of the molecular movement (Le Bihan, 1986). As an example, cerebrospinal fluid will have a high diffusion coefficient because its water molecules can move freely, and anisotropy will be virtually zero, since movement can occur in any direction. In contrast, water molecules that are bound within myelinated axons of the white matter will have a relatively low diffusion coefficient since they can move less freely, and high anisotropy, since the direction of their movement will be restricted to travel along the axis of the axon.

In the presence of a breakdown of myelin or with inflammation, normal barriers to movement of molecules will be diminished, resulting in increases to the diffusion coefficient and decreased anisotropy, since the normal directional diffusion of water molecules will be disrupted (Bammer, 2003). In this way, diffusion measures are particularly sensitive to inflammation and other pathological conditions that affect the integrity of white matter tracts. Like other forms of MRI, this approach also requires careful screening for contraindications for a magnetic field and may produce anxiety or claustrophobic reactions in some subjects.

Issues in image analysis

In addition to developments in scan data acquisition, the development and application of neuroimage analysis techniques is a rapidly growing area of research. Given the substantial effort and expense associated with acquiring high quality neuroimaging data, researchers often apply multiple analysis methods to the same data 
set to address complementary research questions from the potentially rich source of information embedded in the imaging scans. With the ever increasing processing speeds of computer workstations and corresponding developments in computer software programs for image analysis, the available choices for image post-processing and analysis methods have grown significantly in recent years.

\section{Image Post-Processing}

Once the scans are reconstructed to produce brain image data files, postprocessing of the image is typically performed to provide values for subsequent statistical analyses. One approach commonly used for image post-processing has been the region of interest $(\mathrm{ROI})$ method. In the ROI approach individual brain structures or regions are manually traced on each successive slice of a scan using computer software programs designed to display, trace, and store quantitative measures derived from brain image data files. The area of the structure across the traced regions are summed over the slices in a scan and multiplied by the slice thickness to produce a measure of brain tissue volume and the manually defined region can also be used with functional imaging to provide a measure of brain activity for a specific structure or region. The boundaries of the structures and regions are typically defined by strict anatomical landmarks and criterion rules to produce high inter- and intra-rater reliabilities.

In contrast, the voxel-based approach to image post-processing uses automated methods to define brain regions using the smallest volume element in the scan (or voxel), typically in the range of 1 to 10 cubic millimeters in size, depending on the scan 
type (e.g., PET, MRI, fMRI), the image acquisition parameters, and the spatial resolution of the scanner. In this case, computer analysis software are used to align and spatially warp the individual brain scans to a common image template with each voxel in the scan assigned to a three dimensional $x, y$, and $z$ coordinate that allows for both group and within subject comparisons. Each voxel is typically smoothed with a Gaussian filter to enhance the normal distribution of the data for subsequent statistical analysis. In this case, scans obtained from multiple subjects and/or from the same subject under different conditions can be aligned so that between-group and withinsubject statistical analyses can be performed at each voxel and results can be mapped to show statistically significant effects throughout the brain. Although the voxel-based methods have a number of potential advantages over manual tracing, including being non-subjective, highly reliable, and covering the entire brain, the regional accuracy of this approach is dependent on the quality of the spatial warping and alignment algorithms which may not always perform equally well for all subject groups and for all regions of the brain.

In structural imaging of brain tissue volume, the resulting regional measures with ROI or voxel-based methods are usually corrected for subject differences in head size, usually with a traced measure or estimate of the intracranial volume (Raz, 2000). Although tracing methods are quite labor intensive, require a knowledge of neuroanatomy, and involve a significant investment in technician training to achieve high levels of reliability, they have proven to be valuable methods for identifying group differences in regional brain volume, such as those observed in healthy aging (Raz et al., 1998; Sullivan et al., 1995, 2001) and for differentiating the early pathological effects 
of neurodegenerative disorders, like Alzheimer's disease (de Leon et al., 1989; Jack et al., 1992; Killiany et al. 1993; Krasuski et al., 1998, 2002). Since manually traced ROI methods can be quite time-consuming, requiring structures and regions to be traced on each scan slice, this approach may not be practical for very large studies.

In some laboratories, manually traced region of interest measures of volume or function include several additional voxel-based computational analysis steps that are applied to first segment the images into tissue types, prior to identifying structures. Segmentation methods can use one or combine two or more types of MRI scans, such as proton-density and T1 weighted images, or T1 and T2 weighted images, using multivariate statistical methods, in order to classify each voxel in the image as gray matter, white matter, or CSF. An additional classification of "abnormal tissue" can also be included. Combining image types can improve the quality of segmentation, as it capitalizes on the most sensitive tissue contrasts in each type of image, where, for example, brain and CSF boundaries are best differentiated on strongly T2-weighted images, whereas gray and white contrast is most prominent on T1-weighted images. These methods require software programs that can be tailored to a particular set of image types, depending upon the type of scanner that is available and the specific needs of the researcher.

Further, recent developments in computational image analysis techniques have provided methods to obtain ROIs of brain structures using more computationally intensive voxel-based modeling and spatial warping algorithms to extract regional information using highly automated approaches (Fishl and Dale, 2000; Desikan et al., 2006). These voxel-based (semi-) automated methods can decrease the need for 
subjective decisions by the user in assigning boundaries to brain structures during manual tracing and make the analysis for large neuroimaging studies more feasible. Thus, they tend to provide higher inter-rater reliabilities, and once the program is developed, these (semi-) automated methods can be considerably faster than pure manual tracing methods. Although such voxel-based automated approaches are being increasingly used for a variety of applications, the manually traced ROI approach has remained a gold standard for comparison to demonstrate the validity of newly developed analytic methods.

Statistical Methods for Image Analysis

ROI methods produce a relatively small set of regional values that can be extracted for each subject and standard statistical software can be used to perform a full range of statistical analyses. In contrast, voxel-based methods can produce hundreds of thousands of regional values for each scan that require specialized software to perform subsequent statistical analyses. Univariate statistical methods have been most commonly applied for both the ROI and voxel-based approaches, e.g., t-test, ANCOVA, and regression, to test hypotheses about group differences, condition effects, or associations with other subject variables, such as cognitive test scores, genetics, or other health-related factors in both hypothesis-driven and exploratory analyses. Correction for the possibility of identifying spurious regional effects (i.e., Type 1 error) with large numbers of univariate mulitiple comparisons is an especially important issue for voxel-based methods. A number of approaches have been used, including correction for the number of resolution elements in the image, false discovery rate, and 
small volume correction, i.e, predefining a smaller regional volume for voxel-based univariate tests (Genovese et al., 2002; Nichols and Hasayaka, 2003). In addition, there is growing use of permutation and boostrap re-sampling methods to aid in identifying robust and stable regional findings (e.g., Nichols and Holmes, 2002; McIntosh and Lobaugh, 2004; Habeck et al., 2005). Several statistical packages specifically developed for image analysis with ROI and voxel-based post-processing and analysis approaches have been developed that include methods for image display that is essential for reviewing the scans for quality assurance during each step in the postprocessing and analysis. These programs also typically include methods for presentation or display of results to help visualize the observed significant effects. Figure 2 illustrates an example of a brain map showing statistically significant differences between a group of patients with $A D$ and healthy controls using a voxel based univariate approach. A list of some of the most commonly used programs is provided in the selected websites section at the end of this chapter.

Interest in the use of multivariate statistical techniques with neuroimaging data has grown dramatically in recent years and such methods are increasingly being used to characterize the regional patterns or distributed networks of brain responses to stimulation and for group differences in structure and function (Moeller et al., 1987; Habeck et al., 2003; Alexander et al., 1999, 2006; Smith et al., 2006; Grady et al., 2001; McIntosh et al., 1992, 1996; Friston et al., 1993, 2003; McKeown et al., 1998). These latter methods typically assess aspects of the covariance patterns in neuroimaging data to identify regional interactions and their relation to behavior or group membership (Alexander and Moeller, 1994). The multivariate approaches include, for example, 
methods such as the scaled subprofile model (SSM; Moeller et al., 1987) and other principal component-based methods (Friston et al., 1993), independent component analyses (McKeown et al., 1998), structural equation modeling (Mcintosh et al., 1992), dynamic causal modeling (Friston et al., 2003), ordinal trend analysis (Habeck et al., 2005), and partial-least squares analysis (PLS; Mcintosh et al., 1996).

\section{Applications: Aging and Alzheimer's disease}

Neuroimaging methods have been used extensively to investigate the aging brain. These techniques have proven useful in tracking how the brain changes across the normal adult lifespan, investigating the intricate and complex relationships between brain changes and cognition in older adults, and differentiating the normal aging process from pathological conditions such as Alzheimer's disease (AD). In this section, we will briefly review the use of neuroimaging methods for the study of aging and Alzheimer's disease, which also have applications in a wide variety of health psychology research areas.

Anatomical changes associated with normal aging are most often assessed with CT and MRI. CT can provide global measures of brain structure in older adults. For example, by measuring the ventricles and overall global brain size, studies using CT have shown that normal aging is associated with expansion of the cerebral ventricles and generalized enlargement of the cerebral sulci (Freedman et al., 1984, for a review). Further, these brain changes are observed to a greater extent in patient groups with Alzheimer's dementia (DeCarli et al., 1990). 
Using T1 weighted MRI to measure brain anatomy, numerous studies have observed alterations in brain structures in aging and AD. Using an ROI approach to measure MRI volumes in healthy aging, the most consistent regions to show declines are the frontal lobes, but reductions in other brain regions have also been observed, including in temporal, parietal, occipital, and cerebellar regions (for a review, see Raz, 2000). In order to determine the regional effects of aging throughout the brain, Good and colleagues (2001) used voxel-based morphometry with univariate analysis in a sample of 465 individuals assessed with MRI over a wide age range. They found that increasing age was correlated with less gray matter in several regions in cortical and subcortical brain areas, as well as in white matter.

In a recent study of aging using the same voxel-based morphometric method in a small group of healthy adults 22 to 77 years of age, multivariate network analysis was used to identify regionally distributed patterns of gray matter volume in healthy aging (Alexander et al., 2006). In this study, a pattern of regional covariance showing reduced gray matter in frontal, temporal, and cerebellar regions was observed with increasing age. Although reductions in frontal gray matter regions are most consistently observed across studies, atrophy in other brain regions in aging may potentially reflect reductions in connectivity with frontal cortex or the presence of underlying diseases and/or genetic risk for neurodegeneration.

There has also been growing interest in the study of white matter changes in the context of healthy aging (Bartzokis, 2004). Studies using DWMRI have shown reductions in white matter tract integrity with greater age that correlates with cognitive performance (Sullivan et al., 2001; Moseley and Bammer, 2002). 
Studies using MRI to characterize the early and progressive effects of AD on brain anatomy have focused on the development of methods to quantify the changes in brain structures known to be vulnerable to $A D$. In this case, T1-weighted volumetric images are most often used to identify and differentiate brain structures with high spatial resolution. Using an ROI approach to manually-trace brain structures in the medial temporal lobes, including the hippocampus, entorhinal cortex, and amygdala have been performed by a number of research groups to evaluate differences between $A D$ patients and healthy elderly controls, as these structures are known to be preferentially affected by pathology early in AD. Differences between groups of $A D$ patients and healthy elderly have been shown using volumetric ROI measures of the hippocampus, a brain structure important for aspects of memory function and one that has been most consistently studied in AD (de Leon et al., 1989; Jack et al., 1992; Killiany et al., 1993; Krasuski et al., 1998). It has also been shown that these volumetric measures decline with the clinical progression of dementia (Jack et al., 2000). Further, non-demented individuals with increased risk for developing AD have also shown reduced medial temporal lobe volumes compared to healthy controls (Jack et al., 1999; Killiany et al., 2000; Krasuski et al., 2002), supporting the potential for using these methods to aid early detection and track the progression of $A D$ even before the onset of dementia symptoms.

Whole brain MRI scans can be quantitatively compared to sequentially acquired follow up scans over a one or more year interval to compute annual rates of decline in whole brain volume, providing highly reliable and effective methods to evaluate decline in AD patients (Fox et al., 1996, 2000; Chen et al., 2004). 
Since AD prominently affects the cortico-cortical connections important for cognitive functions, there has also been interest in assessing the effects of $A D$ on white matter tracts that serve to connect the cortical association areas of the brain. For example, MRI measurements of the corpus callosum have shown reduced area of subregions defined by manually traced ROIs compared to healthy elderly. Further, these white matter reductions in AD have been associated with PET cerebral glucose metabolism and with neuropsychological performance (Teipel et al., 1999; Hampel et al., 1998).

Diffusion-weighted MRI (DWMRI) may also provide an early neuroimaging marker of $A D$. There is substantial support for inflammatory processes in the brain having an important role in the early development of AD (McGeer et al., 2006). DWMRI is well-suited to measure the effects of inflammation on the brain, as it is sensitive to pathology that alters the integrity of white matter. Several recent studies have investigated changes in DWMRI between $A D$ and healthy controls showing differences in diffusion between groups in regions of the corpus callosum and temporal white matter (Hanyu et al.,1998, 1999, Rose et al., 2000).

Numerous resting state FDG PET imaging studies have demonstrated differences in cerebral metabolism between patients with Alzheimer's dementia and healthy controls (e.g., Minoshima et al., 1995; Silverman et al., 2001; Smith et al., 1992; Alexander et al., 2002). For example, using voxel-based univariate analysis to compare regional cerebral metabolism in a group of $14 \mathrm{AD}$ patients and 34 healthy controls, prominent baseline reductions in the parietotemporal brain regions were observed (figure 2; Alexander et al., 2002). Additional declines in the parietotemporal and frontal 
areas were found when the AD patients were assessed with PET after a one-year interval. Studies of cognitively normal individuals with increased genetic risk for developing AD with the apolipoprotein E e4 allele show reductions and increased rates of decline in cerebral glucose metabolism than e4 non-carriers using the same voxelbased analysis method (Reiman et al., 1996; 2001b). These longitudinal PET studies support the potential for using FDG PET as outcome measures to directly evaluate the effects of $A D$ treatments and prevention therapies on the brain.

Other PET tracers are showing great promise in aiding the ability to detect and directly image the neuropathological and cellular processes in AD in the brain. These include PET ligands to image the regional distribution of amyloid pathology (Agdeppa et al., 2003; Klunk et al., 2003), as well as tracers that assess inflammation and cellular signal transduction (Cagin et al., 2001; Rapoport, 2005). Such approaches provide a complement to the use of FDG with PET, having the potential to enhance understanding of the mechanism of $A D$, as well as to aid early detection, track progression, and potentially evaluate treatments and prevention therapies.

PET studies with FDG and $\mathrm{H}_{2}{ }^{15} \mathrm{O}$ rCBF have also been used to assess regional brain activity in aging and $A D$ during the performance of cognitive tasks and sensory stimulation (Mentis et al., 1996; Pietrini et al., 2000; Grady et al., 2001, 2005). In a study of healthy aging, Cabeza and colleagues (1997) used PET with a multivariate partial least squares analysis to compare rCBF in young (mean 26 years) and older (mean 70 years) adults while they were encoding, recognizing, and recalling a series of word pairs. Young subjects showed greater activation than older adults in left prefrontal and occipito-temporal regions during encoding, and in right prefrontal and parietal regions 
during retrieval. Older adults showed relatively higher activation than young subjects in several regions, including insular cortex during encoding, and left prefrontal regions during retrieval. The results suggest that age is associated with changes in the patterns of neural activity in brain systems underlying memory and may relate to the ability of the brain to functionally compensate for the effects of normal aging.

Such studies have helped to identify regional differences in response to stimulation and task demands in healthy aging and in AD patients, paving the way for the use of fMRI to evaluate how brain systems fail or become reorganized in the context of aging and neurodegenerative disease (e.g., Buckner et al., 2000; Kato et al., 2001; Johnson et al., 2006). There is also growing interest in the use of fMRI to evaluate the effects of treatments and prevention therapies on the neural systems related to specific cognitive or behavioral processes. The use of $\mathrm{fMRI}$ to combine cognitive tasks with pharmacological challenge provides the potential to directly target specific cognitive processes and the associated neural systems for modulation by a specific drug treatment or intervention. In a study by Furey and colleagues (2000), the effect of enhancing the cholinergic neurotransmitter system with a cholinesterase inhibitor during the performance of a face-matching working memory task was investigated in a group of healthy adults. Cholinergic enhancement improved working memory performance and increased activation in brain areas known to be important for the selectivity of perceptual processing during attentional encoding of the task.

An emerging area of research includes the use of neuroimaging systems designed for small animal imaging. With the recent development of transgenic mouse models that produce the amyloid plaque and neurofibrillary tangle pathology similar to 
what is observed in human $A D$, the opportunity to evaluate in vivo brain changes in these animal models provides a potentially important approach for evaluating new treatments and to track brain changes in relation to behavior (Reiman et al., 2001a).

Taken together, neuroimaging research in aging and AD have supported the use of methods such as PET, structural MRI, fMRI, and DWMRI to enhance our understanding of the mechanism of cognitive function and dysfunction, to evaluate patient group differences, to aid early detection of disease effects, to track disease progression from the earliest to end stages of disease, and to potentially evaluate treatments and prevention therapies. Although in this chapter we have focused specifically on applications to the study of aging and Alzheimer's disease, these and other neuroimaging methods have and continue to be applied to an ever-increasing variety of health-related research questions.

\section{Recommended Readings}

Buxton, R.B. (2002). Introduction to functional magnetic resonance imaging: Principles \& techniques. New York, NY: Cambridge University Press.

Frackowiak, R.S.J., Friston, K.J., Frith, C.D., Dolan, R.J., Price, C.J., Zeki, S., Ashburner, J., Penny, W. (2004). Human brain function ( $2^{\text {nd }}$ Edition). Oxford, UK: Elsevier.

\section{Selected Websites for Commonly Used Image Analysis Programs}

Statistical Parametric Mapping (SPM) - http://www.fil.ion.ucl.ac.uk/spm/

Freesurfer - http://surfer.nmr.mgh.harvard.edul

Functional Software Library (FSL) - http://www.fmrib.ox.ac.uk/fsl/

MRIcro - http://www.sph.sc.edu/comd/rorden/mricro.html 
Analysis of Functional Neuroimaging (AFNI) - http://afni.nimh.nih.gov/afni/

Brains - http://www.psychiatry.uiowa.edu/wiki/index.php

Analyze - http://www.mayo.edu/bir/Software/Analyze/Analyze1NEW.html

\section{References}

Agdeppa, E.D., Kepe, V., Liu, J., Small, G.W., Huang, S.C., Petric, A., Satyamurthy, N., \& Barrio, J.R. (2003). 2-Dialkylamino-6-acylmalononitrile substituted naphthalenes (DDNP analogs): novel diagnostic and therapeutic tools in Alzheimer's disease. Mol Imaging Biol, 5, 404-17.

Alexander, G.E., Chen, K., Merkley, T.L., Reiman, E.M., Caselli, R.J., Aschenbrenner, M., Lewis, D.J., Pietrini, P., Teipel, S.J., Hampel, H., Rapoport, S.I., \& Moeller, J.R. (2006). Regional network of magnetic resonance imaging gray matter volume in healthy aging. NeuroReport, 17, 951-6.

Alexander, G.E., Chen, K., Pietrini, P., Rapoport, S.I., \& Reiman, E.M. (2002).

Longitudinal PET evaluation of cerebral metabolic decline in dementia: A potential outcome measure in Alzheimer's disease treatment studies. American Journal of Psychiatry, 159, 738-55.

Alexander, G.E., Mentis, M.J., Van Horn, J.D., Grady, C.L., Berman, K.F., Furey, M.L., Pietrini, P., Schapiro, M.B., Rapoport, S.I., \& Moeller, J.R. (1999). Individual differences in PET activation of object perception and attention systems predict face matching accuracy. NeuroReport, 10, 1965-1971.

Alexander, G.E., \& Moeller, J.R. (1994). Application of the Scaled Subprofile Model to functional imaging in neuropsychiatric disorders: A principal component approach 
to modeling regional patterns of brain function in disease. Human Brain Mapping, 2, 79-94.

Alexander, G.E., Prohovnik, I., Stern, Y., \& Mayeux, R. (1994). WAIS-R subtest profile and cortical perfusion in Alzheimer's disease. Brain and Cognition, 24, 24-43.

Alexander, G.E., Prohovnik, I., Sackeim, H.A., Stern, Y., \& Mayeux, R. (1995). Cortical perfusion and gray-matter weight in frontal lobe dementia. Journal of Neuropsychiatry and Clinical Neurosciences, 7, 188-196.

Ambrose, J., \& Hounsfield, G. (1972a). Computerised transverse axial tomography. British Journal of Radiology, 46,148-149.

Ambrose, J., \& Hounsfield, G. (1972b). Computerised transverse axial scanning (tomography). Part 2: clinical applications. British Journal of Radiology, 46, 19231047.

Bartzokis, G. (2004). Age-related myelin breakdown: A developmental model of cognitive decline and Alzheimer's disease. Neurobiology of Aging, 25, 5-18.

Bammer, R. (2003). Basic principles of diffusion-weighted imaging. European Journal of Radiology, 45, 169-84.

Bandettini, P.A., Wong, E.C., Tikofsky, R.S., Hinks, R.S., \& Hyde, J.S. (1992). Time course EPI of human brain function during task activation. Magn Reson Med, 25, 390-397.

Baron, J.C., Rougemont, D., Soussaline, F., Bustany, P., Crouzel, C., Bousser, M.G., \& Comar, D. (1984). Local interrelationships of cerebral oxygen consumption and glucose utilization in normal subjects and in ischemic stroke patients: A positron tomography study. Journal of Cerebral Blood Flow and Metabolism, 4, 140-9. 
Bloch, F., Hansen, W.W., \& Packard, M. (1946). Nuclear induction. Physics review, 69, 127L, 1946.

Broca, P. (1879). Bull Acad Med (Paris) 2S, 1331-1347.

Buckner, R.L., Snyder, A.Z., Sanders, A.L., et al. (2000). Functional brain imaging of young, nondemented, and demented older adults. J Cogn Neurosci, 12 Suppl 2, 24-34.

Busatto, G.F., \& Pilowsky, L.S. (1995). Neuroreceptor mapping with in-vivo imaging techniques: Principles and applications. Br J Hospl Med, 53, 309-13.

Cabeza, R., Grady, C.L., Nyberg, L., McIntosh, A.R., Tulving, E., Kapur, S., Jennings, J.M., Houle, S., \& Craik, F.I.M. (1997). Age-Related Differences in Neural Activity during Memory Encoding and Retrieval: A Positron Emission Tomography Study. Journal of Neuroscience, 17, 391-400.

Cagnin, A., Brooks, D.J., Kennedy, A.M., et al. (2001). In-vivo measurement of activated microglia in dementia. Lancet, 358, 461-7.

Chen, K., Reiman, E.M., Alexander, G.E., Bandy, D., Renaut, R., Crum, W.R., Fox, N.C., \& Rossor, M.N. (2004). An automated algorithm for the computation of brain volume change from sequential MRI's using an iterative principal component analysis and its evaluation for the assessment of whole brain atrophy rates in patients with probable Alzheimer's disease. Neuroimage, 22, 134-43.

DeCarli, C., Kaye, J.A., \& Horwitz, B. (1990). Critical analysis of the use of computer assisted transverse axial tomography to study human brain in aging and dementia of the Alzheimer type. Neurology, 40, 872-883. 
de Leon, M.J., George, A.E., Stylopoulos, L.A., et al. (1989). Early marker for Alzheimer's disease: The atrophic hippocampus. Lancet, 2, 672-673.

Desikan, R.S., Segonne, F., Fischl, B., Quinn, B.T., Dickerson, B.C., Blacker, D., Buckner, R.L., Dale, A.M., Maguire, R.P., Hyman, B.T., Albert, M.S., \& Killiany, R.J. (2006). An automated labeling system for subdividing the human cerebral cortex on MRI scans into gyral based regions of interest. Neuroimage, 31, 968-80.

Fischl, B., \& Dale, A.M. (2000). Measuring the thickness of the human cerebral cortex from magnetic resonance images. Proc Natl Acad Sci U S A, 97, 11050-5.

Fox, N.C., Cousens, S., Scahill, R., Harvey, R.J., \& Rossor, M.N. (2000). Using serial registered brain magnetic resonance imaging to measure disease progression in Alzheimer disease: power calculation and estimates of sample size to detect treatment effects. Arch Neurol, 57, 339-44.

Fox, N.C., Freeborough, P.A., \& Rossor, M.N. (1996). Visualisation and quantification of rates of atrophy in Alzheimer's disease. Lancet, 348, 94-97.

Fox, P., \& Raichle, M.E. (1986). Focal physiological uncoupling of cerebral blood flow and oxidative metabolism during somatosensory stimulation in human subjects. Proc Natl Acad Sci USA, 83,1140-1144.

Frackowiak, R.S.J., Lenzi, G.L., Jones, T., \& Heather, J.D. (1980). Quantitative measurement of regional cerebral blood flow and oxygen metabolism in man using 150 and positron emission tomography: theory, procedure and normal values. J Comput Assist Tomogr, 4, 727-736. 
Friston, K.J., Frith, C., Liddle, F.P., \& Frackowiak, R.S.J. (1993). Functional connectivity: The principal-component analysis of large (PET) data sets. Journal of Cerebral Blood Flow and Metabolism, 13, 5-14.

Friston, K.J. (2003). Functional connectivity. In R.S.J. Frackowiak, K.J. Friston, C. Frith, R. Dolan, C.J. Price, S. Zeki, J. Ashburner, and W.D. Penny, (Eds), Human Brain Function. Academic Press, 2nd edition.

Freedman, M., Knoefel, J., Naeser, M., Levine, H. (1984). Computerized axial tomography in aging. In M.L. Albert (Ed), Clinical neurology of aging, pp. 139-148, New York: Oxford University Press.

Furey, M.L., Pietrini, P., \& Haxby, J.V. (2000). Cholinergic enhancement and increased selectivity of perceptual processing during working memory. Science, 290, 2315-9. Genovese, C.R., Lazar, N., \& Nichols, T.E. (2002). Thresholding of statistical maps in functional neuroimaging using the false discovery rate. Neuroimage, 15, 870-878.

Good, C.D., Johnsrude, I.S., Ashburner, J., Henson, R.N., Friston, K.J., \& Frackowiak, R.S. (2001). A voxel-based morphometric study of ageing in 465 normal adult human brains. Neuroimage, 14, 21-36.

Grady, C.L., Mclntosh, A.R., \& Craik, F.I. (2005). Task-related activity in prefrontal cortex and its relation to recognition memory performance in young and old adults. Neuropsychologia, 43, 1466-81.

Grady, C.L., Furey, M.L., Pietrini, P., Horwitz, B., \& Rapoport, S.I. (2001). Altered brain functional connectivity and impaired short-term memory in Alzheimer's disease. Brain, 124, 739-56. 
Habeck, C., Hilton, H.J., Zarahn, E., Flynn, J., Moeller, J., \& Stern, Y. (2003). Relation of cognitive reserve and task performance to expression of regional covariance networks in an event-related fMRI study of nonverbal memory. Neuroimage, 20, 1723-33.

Habeck, C., Krakauer, J.W., Ghez, C., Sackeim, H.A., Eidelberg, D., Stern, Y., \& Moeller, J.R. (2005). A new approach to spatial covariance modeling of functional brain imaging data: ordinal trend analysis. Neural Comput, 17, 1602-45.

Hampel, H., Teipel, S.J., Alexander, G.E., Horwitz, B., Teichberg, D., Schapiro, M.B., \& Rapoport, S.I. (1998). Corpus callosum atrophy is a possible indicator of regionand cell type-specific neuronal degeneration in Alzheimer disease: a magnetic resonance imaging analysis. Arch Neurol, 55, 193-8.

Hanyu, H., Sakurai, H., Iwamoto, T., Takasaki, M., Shindo H., \& Abe K. (1998).

Diffusion-weighted MR imaging of the hippocampus and temporal white matter in Alzheimer's disease. Journal of Neurological Sciences, 156,195-200.

Hanyu, H., Asano, T., Sakurai, H., Imon, Y., Iwamoto, T., Takasaki, M., Shindo, H., \& Abe, K. (1999). Diffusion-weighted and magnetization transfer imaging of the corpus callosum in Alzheimer's disease. Journal of the Neurological Science, 167, 37-44.

Jack, C.R., Petersen, R.C., O'Brien, P., et al. (1992). MR-based hippocampal volumetry in the diagnosis of Alzheimer's disease. Neurology, 42, 183-8.

Jack, C.R., Petersen, R.C., Xu, Y.C., et al. (1999). Prediction of AD with MRI-based hippocampal volume in mild cognitive impairment. Neurology, 52, 1397-1403. 
Jack, C.R., Petersen, R.C., Xu, Y., et al. (2000). Rates of hippocampal atrophy correlate with change in clinical status in aging and AD. Neurology, 55, 484-9.

Jagust, W.J., Eberling, J.L., Richardson, B.C., et al. (1993). The cortical topography of temporal lobe hypometabolism in early Alzheimer's disease. Brain Res, 629, 18998.

Johnson, S.C., Schmitz, T.W., Moritz, C.H., Meyerand, M.E., Rowley, H.A., Alexander, A.L., Hansen, K.W., Gleason, C.E., Carlsson, C.M., Asthana, S., Chen, K., Reiman, E.M., \& Alexander, G.E. (2006). Activation of brain regions vulnerable to Alzheimer's disease: The effect of mild cognitive impairment. Neurobiology of Aging, 27, 1604-12.

Kato, T., Knopman, D., \& Liu, H. (2001). Dissociation of regional activation in mild AD during visual encoding: a functional MRI study. Neurology, 57, 812-6.

Kety, S.S., \& Schmidt, C.F. (1945). The determination of cerebral blood flow in man by the use of nitrous oxide in low concentrations. American Journal of Physiology, 143, 53-66.

Klunk, W.E., Engler, H., Nordberg, A., Bacskai, B.J., Wang, Y., Price, J.C., Bergstrom, M., Hyman, B.T., Langstrom, B., \& Mathis, C.A. (2003). Imaging the pathology of Alzheimer's disease: amyloid-imaging with positron emission tomography. Neuroimaging Clin N Am,13, 781-9.

Killiany, R.J., Gomez-Isla, T., Moss, M., et al. (2000). Use of structural magnetic resonance imaging to predict who will get Alzheimer's disease. Ann Neurol, 47, 430-9. 
Killiany, R.J., Moss, M.B., Albert, M.S., Sandor, T., Tieman, J., \& Jolesz, F. (1993). Temporal lobe regions on magnetic resonance imaging identify patients with early Alzheimer's disease. Arch Neurol, 50, 949-54.

Krasuski, J.S., Alexander, G.E., Daly, E., Murphy, D.C.M., Rapoport, S.I., \& Schapiro, M.B. (1998). Volumes of medial temporal lobe structures in patients with Alzheimer's disease and mild cognitive impairment (and in healthy controls). Biological Psychiatry, 43, 60-68.

Krasuski, J.S., Alexander, G.E., Horwitz, B., Rapoport, S.I., \& Schapiro, M.B. (2002). Relation of medial temporal lobe volumes to age and memory function in nondemented Down syndrome adults: Implications for the prodromal phase of Alzheimer's disease. American Journal of Psychiatry, 159, 74-81.

Lassen, N.A., Hoedt-Rasmussen, K., Sorensen, S.C., Skinhoj, E., Cronquist, B., Bodforss, E., \& Ingvar, D.H. (1963). Regional cerebral blood flow in man determined by Krypton-85. Neurology, 13, 719-727.

Lauterbur, P. (1973). Image Formation by Induced Local Interactions: Examples Employing Nuclear Magnetic Resonance. Nature 242, 190-1.

Le Bihan, D., Breton, E., Lallemand, D., Grenier, P., Cabanis, E., \& Laval-Jeantet, M. (1986). MR imaging of intravoxel incoherent motions: application to diffusion and perfusion in neurologic disorders. Radiology, 161, 401-7.

Mansfield, P. (1976). Multiplanar image formation using NMR spin echoes. J Phys C Solid State Phys, 10, L55-8. 
McGeer, P.L., Rogers, J., \& McGeer, E.G. (2006). Inflammation, anti-inflammatory agents and Alzheimer disease: the last 12 years. J Alzheimers Dis, 9(3 Suppl), 271-6.

McIntosh, A.R., Bookstein, F.L., Haxby, J.V., \& Grady, C.L. (1996). Spatial pattern analysis of functional brain images using partial least squares. Neuroimage, 3 , 143-57.

McIntosh, A. R., \& Gonzalez-Lima, F. (1992). The application of structural modeling to metabolic mapping of functional neural systems. In: F. Gonzalez-Lima, T.

Finkenstädt, and H. Scheich (Eds). Advance in Metabolic Mapping Techniques for Brain Imaging of Behavioral and Learning Functions. Kluwer Academic Publishers, Dordrecht. pp 219-255.

McIntosh, A.R., \& Lobaugh, N.J. (2004). Partial least squares analysis of neuroimaging data: applications and advances. Neuroimage, 23 Suppl 1, S250-63.

McKeown, M. J., Makeig, S., Brown, G. G., Jung, T., Kindermann, S. S., Bell, A. J., \& Sejnowski, T. J. (1998). Analysis of fMRI by blind separation into independent spatial components. Human Brain Mapping, 6, 160-188.

Mentis, M.J., Horwitz, B., Grady, C.L., Alexander, G.E., VanMeter, J.W., Maisog, J.Ma., Pietrini, P., Schapiro, M.B., \& Rapoport, S.I. (1996). Visual cortical dysfunction in Alzheimer's disease evaluated with a temporally graded "stress test" during PET. American Journal of Psychiatry, 153, 32-40.

Minoshima, S., Frey, K.A., Koeppe, R.A., et al. (1995). A diagnostic approach in Alzheimer's disease using three-dimensional stereotactic surface projections of Fluorine-18-FDG PET. J Nucl Med, 36, 1238-48. 
Moeller, J.R., Strother, S.C., Sidtis, J.J., \& Rottenberg, D.A. (1987). Scaled subprofile model: A statistical approach to the analysis of functional patterns in positron emission tomographic data. J Cereb Blood Flow Metab, 7, 649-658.

Moseley, M., Bammer, R., \& Illes, J. (2002). Diffusion-tensor imaging of cognitive performance. Brain Cogn, 50, 396-413.

Nichols, T.E., \& Hayasaka, S. (2003). Controlling the familywise error rate in functional neuroimaging: a comparative review. Statistical Methods in Medical Research, 12, 419-446.

Nichols, T.E., \& Holmes, A.P. (2002). Nonparametric permutation tests for functional neuroimaging: a primer with examples. Human Brain Mapping, 15,1-25.

Obrist, W.D., Thompson, H.K., King, C.H., et al. (1967). Determination of regional cerebral blood flow by inhalation of 133-xenon. Circul Res, 20, 124-35.

Ogawa, S., \& Lee, T.M. (1990). Magnetic resonance imaging of blood vessels at high fields: In vivo and in vitro measurements and image simulation. Magnetic Resonance in Medicine, 16, 9-18.

Pietrini, P., Furey, M.L., Alexander, G.E., Dani, A., Mentis, M.J., Freo, U., Horwitz, B., Rapoport, S.I., \& Schapiro, M.B. (2000). Brain synaptic efficiency progressively declines in Alzheimer's disease: A PET-FDG stimulation study. Journal of Nuclear Medicine, 41, 575-83.

Posner, M.I. \& Raichle M.E. (1994). Images of mind. New York: Scientific American Library WH Freeman \& Company. 
Prohovnik, I. (1988). Data quality, integrity, and interpretation. In S. Knezevic, V.A., Maximilian, Z. Mubrin, I. Prohovnik, and J. Wade (Eds), Handbook of regional cerebral blood flow, pp.51-77. Hillsdale, NJ: Lawrence Erlbaum Associates, Inc.

Prohovnik, I., Smith, G., Sackeim, H.A., Mayeux, R., \& Stern, Y. (1989). Gray-matter degeneration in presenile Alzheimer's disease. Ann Neurol, 25, 117-24.

Purcells, E., Torrey, H.C., \& Pound, R.V. (1946). Resonance absorption by nuclear magnetic moments in a solid. Phys Rev, 69, 37-38.

Rapoport, S.I. (2005). In vivo approaches and rationale for quantifying kinetics and imaging brain lipid metabolic pathways. Prostaglandins Other Lipid Mediat, 77,18596.

Raz, N. (2000). Aging of the brain and its impact on cognitive performance: Integration of structural and functional findings. In: F.I.M. Craik and T.A. Salthouse (Eds) Handbook of Aging and Cognition - II, pp. 1-90. Mahwah, NJ : Erlbaum.

Raz, N., Gunning-Dixon, F.M., Head, D., Dupuis, J.H., \& Acker, J.D. (1998). Neuroanatomical correlates of cognitive aging: evidence from structural magnetic resonance imaging. Neuropsychology, 12, 95-114.

Reiman, E.M., Caselli, R.J., Yun, L.S., Chen, K., Bandy, D., Minoshima, S., Thibodeau, S.N., \& Osborne, D. (1996). Preclinical evidence of Alzheimer's disease in persons homozygous for the epsilon 4 allele for apolipoprotein E. N Engl J Med, 334, 7528.

Reiman, E.M., Caselli, R.J., Alexander, G.E., \& Chen, K. (2001a). Tracking the decline in cerebral glucose metabolism in persons and laboratory animals at genetic risk for Alzheimer's disease. Clin Neurosci Res, 1, 194-206. 
Reiman, E.R., Caselli, R.J., Chen, K., Alexander, G.E., Bandy, D., \& Frost, J. (2001b). Declining brain activity in cognitively normal apolipoprotein E e4 heterozygotes: A foundation for using positron emission tomography to efficiently test treatments to prevent Alzheimer's disease. Proceedings of the National Academy of Sciences USA, 98, 3334-9.

Risberg, J. (1980). Regional cerebral blood flow measurements by 133-Xe-inhalation: methodology and application in neuropsychology and psychiatry. Brain and Language, 9, 9-34.

Rose, S.E., Chen, F., Chalk, J.B., Zelaya, F.O., Strugnell, W.E., Benson, M., Semple, J., \& Doddrell, D.M. (2000). Loss of connectivity in Alzheimer's disease: an evaluation of white matter tract integrity with colour coded MR diffusion tensor imaging. Journal of Neurology, Neurosurgery \& Psychiatry, 69(4), 528-30.

Silverman, D.H.S., Small, G.W., Chang, C.Y., Lu, C.S., Kung de Aburto, M.A., Chen, W., Czernin, J., Rapoport, S.I., Pietrini, P., Alexander, G.E., Schapiro, M.B., Jagust, W.J., Hoffman, J.M., Welsh-Bohmer, K.A., Alavi, A., Clark, C.M., Salmon, E., de Leon, M.J., Mielke, R., Cummings, J.L., Kowell, A.P., Gambhir, S.S., Hoh, C.K., \& Phelps, M.E. (2001). Neuroimaging in evaluation of dementia: Regional brain metabolism and long-term outcome. Journal of the American Medical Association, 286, 2120-7.

Smith, G.S., de Leon, M.J., George, A.E., Kluger, A., Volkow, N.D., McRae, T., Golomb, J., Ferris, S.H., Reisberg, B., Ciaravino, J., et al. (1992). Topography of crosssectional and longitudinal glucose metabolic deficits in Alzheimer's disease. Pathophysiologic implications. Arch Neurol, 49(11),1142-50. 
Smith, J.F., Chen, K., Johnson, S.C., Morrone-Strupinsky, J., Reiman, E.M., Nelson, A., Moeller, J.R., \& Alexander, G.E. (2006). Network analysis of single-subject fMRI during a finger opposition task. Neuroimage, 32, 325-32.

Sokoloff, L. (1982). The radioactive deoxyglucose method, theory, procedure and application for the measurement of local cerebral glucose utilization in the central nervous system. In BW Agranoff and MH Aprison (Eds), Advances in Neurochemistry, vol 4, pp 1-82. New York: Plenum Publishing Corporation.

Sullivan, E.V., Adalsteinsson, E., Hedehus, M., Ju, C., Moseley, M., Lim, K.O., \& Pfefferbaum, A. (2001). Equivalent disruption of regional white matter microstructure in ageing healthy men and women and women. Neuroreport, 12, 99-104.

Sullivan, E.V., Marsh, L., Mathalon, D.H., Lim, K.O., \& Pfefferbaum, A. (1995). Agerelated decline in MRI volumes of temporal lobe gray matter but not hippocampus. Neurobiol Aging,16, 591-606.

Teipel, S.J., Hampel, H., Pietrini, P., Alexander, G.E., Horwitz, B., Daly, E., Schapiro, M.B., Moller, H-J., \& Rapoport, S.I. (1999). Region specific corpus callosum atrophy correlates with regional pattern of cortical glucose metabolism in Alzheimer's disease. Archives of Neurology, 56, 467-473.

Young, J.R., Hall, A.S., Pallis, C.A., Legg, N.J., Bydder, G.M., \& Steiner, R.E. (1981). Nuclear magnetic resonance imaging of the brain in multiple sclerosis. Lancet , 2, 1063-66. 